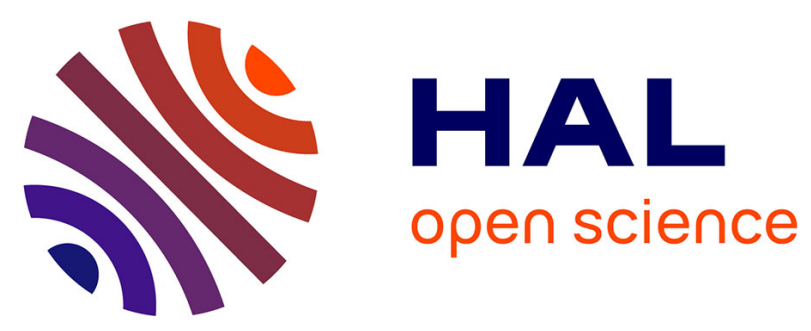

\title{
La microholographie: une technique pour la mesure des positions, des tailles et des vitesses des particules présentes dans un écoulement
}

\author{
M. Moine, C. Ducottet, M. Courbon, T. Fournel
}

\section{- To cite this version:}

M. Moine, C. Ducottet, M. Courbon, T. Fournel. La microholographie: une technique pour la mesure des positions, des tailles et des vitesses des particules présentes dans un écoulement. Journal de Physique IV Proceedings, 1994, 04 (C4), pp.C4-717-C4-717. 10.1051/jp4:19944195 . jpa-00252656

\section{HAL Id: jpa-00252656 https://hal.science/jpa-00252656}

Submitted on 1 Jan 1994

HAL is a multi-disciplinary open access archive for the deposit and dissemination of scientific research documents, whether they are published or not. The documents may come from teaching and research institutions in France or abroad, or from public or private research centers.
L'archive ouverte pluridisciplinaire HAL, est destinée au dépôt et à la diffusion de documents scientifiques de niveau recherche, publiés ou non, émanant des établissements d'enseignement et de recherche français ou étrangers, des laboratoires publics ou privés. 


\title{
La microholographie : une technique pour la mesure des positions, des tailles et des vitesses des particules présentes dans un écoulement
}

\author{
M. MOINE, C. DUCOTTET, M. COURBON et T. FOURNEL
}

Laboratoire Traitement du Signal et Instrumentation, URA 842 du CNRS, Université Jean Monnet, 23 rut du Docteur Paul Michelon, 42023 Saint Etienne cedex 02, France

Un champ de petites particules ( 5 à $100 \mu$ ) en suspension dans un écoulement à un instant donné peut être enregistré sur un hologramme. Cet hologramme est une prise de vue des franges résultant des intertérences entre le faisceau d'un laser à impulsion éclairant l'écoulement et la lumière rediffusée par les particules. II est possible de restituer ce champ tridimensionnel en éclairant l'hologramme avec une source cohérente continue. L'utilisation de plusieurs impulsions lors de l'enregistrement permet la détermination des vitesses des particules.

Le dépouillement de l'hologramme se fait par déplacement d'une caméra dans le champ restitué. Le but du travail présenté est de repérer de façon automatique les positions de particules. Cette opération est rendue délicate par la présence de speckle dont l'intensité locale peut être du même ordre que celle des particules au voisinage de leur plan focal.

Le repérage des particules au milieu du speckle est réalisé par la détection automatique des anneaux apparaissant sur des plans proches de la position de mise au point des particules. Une analyse aussi bien théorique qu'expérimentale de l'ensemble du processus est donnée. Les résultats de l'analyse théorique permettent, en outre, l'estimation de la distance au plan focal de la particule.

Les champs de vitesse expérimentaux tridimensionnels sont importants en Mécanique des Fluides pour ajuster les modèles théoriques. L'évolution des structures turbulentes dans un écoulement n'est pas encore bien comprise. 